\title{
Compartimentalización de la biomasa aérea de Grevillea robusta A. en función a la densidad inicial de plantación
}

\author{
Aboveground biomass allocation of Grevillea robusta A. \\ based on initial planting density
}

\author{
Sara Regina Barth¹, Ana María Giménez², Marisa Jacqueline Joseau³, \\ María Elena Gauchat ${ }^{4}$ e Hugo Enrique Fassola ${ }^{4}$
}

\begin{abstract}
Resumo
A densidade de plantação a uma determinada idade é um indicador confiável da ocupação arbórea de um local, representando assim a estrutura das florestas. O objetivo deste estudo foi avaliar o efeito da densidade de plantio inicial no acúmulo da biomassa de plantações de Grevillea robusta a os 18 anos de idade. 0 ensaio foi conduzido em Posadas Misiones, Argentina, com 4 densidades de plantio iniciais (162, 375, 750 e 1500 plantas / ha). O acúmulo da biomassa foi analisado nos estratos arbustivo, herbáceo, piso florestal e arbóreo (folhas, galhos, tronco, casca). Nas árvores é considerado tanto o efeito sobre os indivíduos e sobre o sítio. A biomassa total do sub-bosque apresentou diferenças significativas entre os tratamentos. A distribuição da biomassa variou resultando em aumento da presença de galhos mortos e biomassa do estrato arbustivo em densidades de plantio superiores. A acumulação da biomassa herbácea foi maior no tratamento da plantação da menor densidade. O maior espaçamento resultou num maior diâmetro dos indivíduos, mas a maior acumulação da biomassa do fuste por hectare foi em espaçamentos menores.
\end{abstract}

Palavras-chave: Distribuição da biomassa, sub-bosque, estrato arbóreo, espaçamento, manejo florestal.

\begin{abstract}
Planting density at a given age is a reliable indicator of the degree of a site's arboreal cover and thus it represents the structure of the forest stand. The objective of this paper was to assess the initial planting density's effect on aboveground biomass accumulation in an 18-year old Grevillea robusta plantation. The test was conducted in Posadas, Misiones (Argentina), with 4 initial planting densities (162, 375, 750 and 1500 plants/ha). Biomass accumulation was analyzed in the shrub, herbaceous, forest floor and trees strata (leaves, branches, stem and bark). In the case of trees, the effects of the treatments were studied in individuals and in the stands. The total understory biomass presented significant differences among treatments. Biomass allocation varied leading to a higher presence of dead branches and shrub biomass at higher planting densities. The herbaceous stratum had a higher biomass accumulation with the lowest planting density treatment. Larger spacing resulted in larger individual diameter but the highest stem biomass accumulation per hectare was observed with the smallest spacing.
\end{abstract}

Keywords: Biomass allocation, understory, arboreal stratum, plant spacing, forest management.

\section{INTRODUCCIÓN}

Grevillea robusta A. se origina en Australia, donde crece en pequeños rodales de los estados de Queensland y Nueva Gales del Sur, en latitudes desde los $25^{\circ} 50^{\prime} \mathrm{S}$ hasta los $30^{\circ} 10^{\prime} \mathrm{S}$, en altitudes desde el nivel del mar hasta los $1100 \mathrm{~m}$. En esa zona las lluvias van desde 720 a $1710 \mathrm{~mm}$ anuales y la temperatura media anual oscila de 14 a $20^{\circ} \mathrm{C}$ (QUEENSLAND FOREST SERVICE, 1928; BOLAND, 1984; HARWOOD; GETAHUN, 1990; HARWOOD, 1992). Su madera se emplea para fabri-

\footnotetext{
${ }^{1}$ M. Sc. Forestales. Instituto Nacional de Tecnología Agropecuaria. Estación Experimental Agropecuaria Montecarlo / Facultad de Ciencias Forestales - Universidad Nacional de Misiones. Avenida El Libertador 2472 - C. P. 3384 - Montecarlo, Misiones, Argentina. E-mail: barth.sara@inta.gob.ar.

${ }^{2}$ Facultad de Ciencias Forestales - Universidad Nacional de Santiago del Estero. Av. Belgrano Sur 1912, 4200 - Santiago del Estero, Argentina. E-mail: amig@unse.edu.ar.

${ }^{3}$ Facultad de Ciencias Agrarias - Universidad Nacional de Córdoba. Av. Haya de la Torre s/n - Córdoba, Argentina. E-mail: jajoseau@agro.unc.edu.ar.

${ }^{4}$ Instituto Nacional de Tecnología Agropecuaria. Estación Experimental Agropecuaria Montecarlo / Facultad de Ciencias Forestales - Universidad Nacional de Misiones. Avenida El Libertador 2472 - C. P. 3384 - Montecarlo, Misiones, Argentina. E-mail: fassola.hugo@inta.gob.ar; gauchat.maria@inta.gob.ar.
}

Sci. For., Piracicaba, v. 44, n. 111, p. 653-664, set. 2016 DOI: dx.doi.org/10.18671/scifor.v44n111.11 
car botes e instrumentos musicales y en ebanistería, tanto de muebles como cerramientos interiores y exteriores, ya que es muy resistente a la humedad. Fue introducida con éxito en numerosos países como Brasil, Argentina, India, Ruanda y Kenia por su potencial de crecimiento y usos: sombreamiento de cultivos agrícolas y pasturas así como leña y madera (GONÇALVES MARTINS; NEVES, 2004; BARTH et al., 2015). Respecto a su cultivo, en su etapa juvenil puede aguantar condiciones algo sombreadas, pero prefiere pleno sol. Se adapta a una gran diversidad de climas y suelos. Grevillea robusta se desarrolla mejor en suelos con $\mathrm{pH}$ ácido o neutro. Su parte subterránea crece con vigor en suelos de textura arenosa, franca o arcillosa, éstos se pueden mantener generalmente secos o húmedos. Soporta bien la sequía y no tolera los encharcamientos. Es una especie medianamente exigente en cuanto a necesidades lumínicas, pudiendo implantarse indistintamente con semisombra o a cielo abierto con exposición solar directa.

Si bien Grevillea robusta es una especie de gran potencial aún no es empleada a gran escala en la industria maderera. Se ignora el efecto del manejo silvícola en el producto a obtener. Dado los conocimientos existentes, ningún ordenador forestal discutiría el hecho de que los espaciamientos mayores favorecen una mayor dimensión de las trozas maderables. No obstante, no se sabe el efecto que dichos espaciamientos tienen en la compartimentalización de la biomasa aérea de Grevillea robusta y en el sotobosque generado, siendo éste el objetivo principal del presente trabajo. La hipótesis planteada fue que la estructura de la masa forestal varía según el manejo silvícola adoptado.

La masa forestal es un importante almacenador de Carbono. Éste se acumula en la biomasa de sus diferentes compartimentos (DIDION et al., 2014).

La biomasa, materia orgánica generada por las plantas como resultado de la actividad fotosintética, es de suma importancia para determinar la productividad de un ecosistema. Por lo tanto, el conocimiento del desempeño de la especie en cuanto a la acumulación de biomasa y sus vinculaciones al crecimiento y calidad de madera obtenida, es una herramienta fundamental para la toma de decisiones. La acumulación de carbono está influenciada principalmente por factores físicos, edáficos y por patrones de disturbio que afectan la estructura y las reservas de biomasa y carbono en la masa forestal. El manejo forestal puede producir cambios en el balance del Carbono (NAVE et al., 2010; JANDL et al., 2011). Alteraciones en el uso de los recursos (luz, agua y nutrientes) afectan tanto al estrato arbóreo como al sotobosque (MARILOU et al., 2004). Según Herrera Arango et al. (2001) este efecto dependerá del área basal del estrato arbóreo.

El crecimiento y la calidad de madera, producto del manejo forestal, se halla vinculado a un incremento de copa. Al aumentar o disminuir la densidad arbórea en una determinada plantación se presentan diferentes comportamientos como consecuencia de la competencia por nutrientes e insumos, lo que produce variaciones en la actividad fotosintética de los individuos (ROCHA; DELLA LUCIA, 1987; ZOBEL; VAN BUIJTENEN, 1989; ACHIM et al., 2006; TEOBALDELLI et al., 2009; BEAULIEU et al., 2011; PRASAD et al., 2011; WANG et al., 2011).

Estimar la variación en la acumulación de biomasa en plantaciones es una herramienta útil en la valoración cuantitativa del Carbono almacenado en las estructuras vivas y muertas en un momento dado. Dicho conocimiento es de vital importancia para evaluar su contribución al ciclo del Carbono, dada las implicancias de la presencia de $\mathrm{CO}_{2}$ en el cambio climático global. Surgió así el interés en conocer la tasa y los patrones de partición de la biomasa en los distintos compartimentos del árbol (ADEGBIDI et al., 2002).

\section{MATERIALES Y MÉTODOS}

El estudio fue realizado con datos recolectados en un ensayo de densidades iniciales de plantación (tabla 1) de Grevillea robusta instalado por INTA en el año 1994 en vinculación con la empresa Danzer forestaciones S. A., actualmente Garruchos Forestal S. A. en Posadas, zona Sur de la Provincia de Misiones, Argentina (figura 1). Las coordenadas del ensayo son $27^{\circ} 29^{\prime} 4,68^{\prime \prime}$ L S y $55^{\circ} 56^{\prime} 48,45^{\prime \prime} \mathrm{L} \mathrm{O}$.

Todos los tratamientos (densidades de plantación) sufrieron 4 podas con remoción de $30 \%$ de copa hasta llegar a una altura de base de copa verde mínima de 4 metros. 
Tabla 1. Espaciamiento y densidades iniciales de plantación del ensayo de Grevillea robusta instalado en Posadas, Misiones, Argentina.

Table 1. Spacing and initial planting densities at the Grevillea robusta trial set up in Posadas, Misiones, Argentina.

\begin{tabular}{cc}
\hline Espaciamiento $(\mathbf{m})$ & Densidad (plantas/hectárea) \\
\hline $7,9 \times 7,85$ & 162 \\
$5,2 \times 5,10$ & 375 \\
$3,7 \times 3,60$ & 750 \\
$2,5 \times 2,65$ & 1500 \\
\hline
\end{tabular}

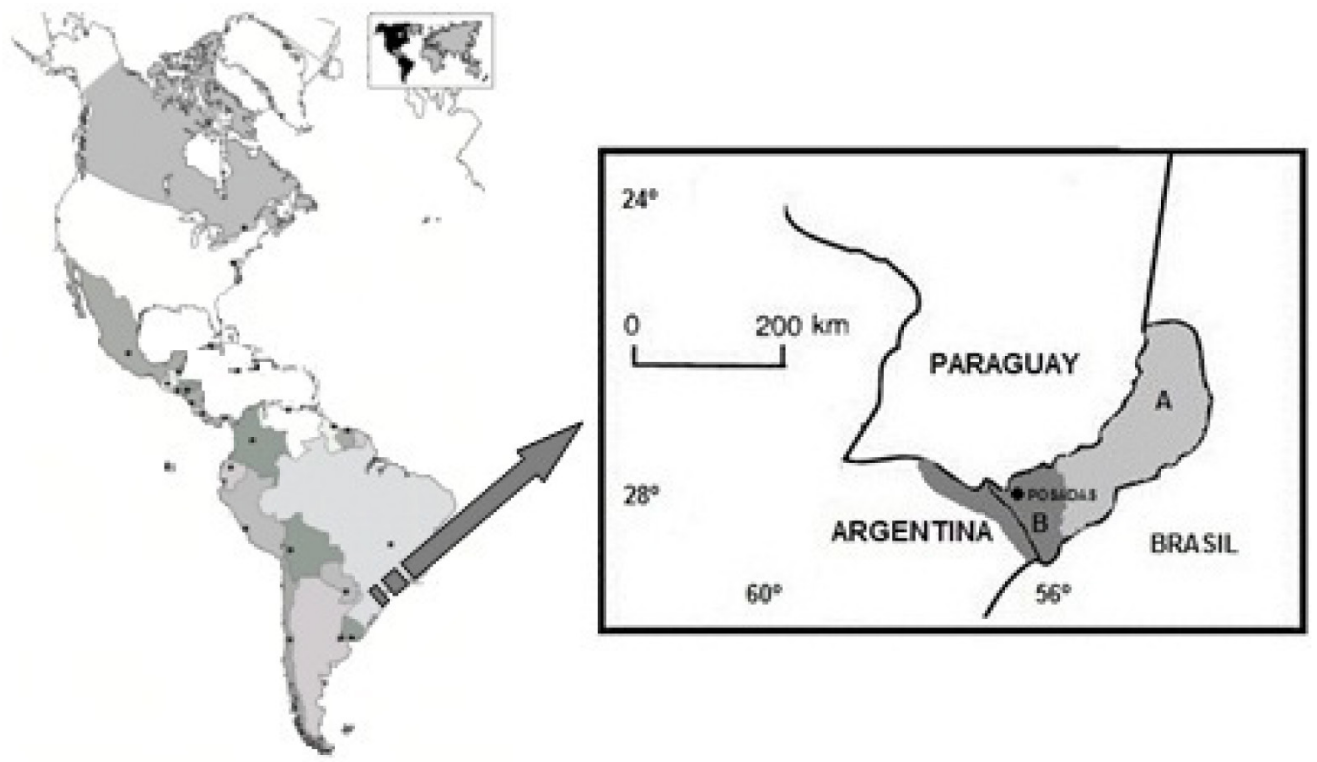

Figura 1. Localización del área de estudio. Referencias: A, Distrito de las Selvas Mixtas; B, Distrito de los Campos. Figure 1. Location map of the study area. References: A, Mixed Forests District; B, Fields Disctrict.

Posadas, pertenece a la Selva Paranaense, Distrito de los Campos (CABRERA, 1976). Este distrito abarca la zona suroeste de la provincia de Misiones y noreste de Corrientes, Argentina, donde surge el ecotono con la Provincia Chaqueña (figura 1). El clima no difiere mucho del Distrito de las Selvas Mixtas (zona centro y norte de Misiones) con suelos lateríticos en ambos distritos. No obstante, las precipitaciones son ligeramente menores, 1800 versus $2000 \mathrm{~mm}$ año (BURGOS, 1970), con una sequía invernal más marcada, que junto a otras características dan lugar a diferencias notorias en la vegetación preponderante. La vegetación predominante en esta zona son los llamados campos o sabanas de gramíneas. La selva solo aparece a orillas de ríos y arroyos y en pequeñas porciones o islas en lugares de mayor humedad del suelo. Las características del suelo se presentan en la tabla 2. Los valores de $\mathrm{pH}$ indican que son suelos fuertemente ácidos, lo que queda reflejado en las bajas concentraciones de P disponible en el suelo. La densidad aparente del suelo se comporta de manera inversa a lo esperado, presentándose los mayores valores de densidad en superficie y disminuyendo con la profundidad debido al uso ganadero anterior.

Tabla 2. Densidad aparente y contenido de nutrientes del suelo a distintas profundidades. Table 2. Bulk density and soil nutrient content at different depths.

\begin{tabular}{|c|c|c|c|c|c|c|c|c|}
\hline espesor & $\begin{array}{c}\text { densidad aparente } \\
\text { Mg.m }{ }^{-3}\end{array}$ & $\mathrm{pH}$ & $\begin{array}{c}\text { materia orgánica } \\
\% \\
\end{array}$ & $\mathrm{~N}$ total & $\mathbf{P}$ & $\begin{array}{c}\text { K } \\
\text { Kg.ha-1 } \\
\end{array}$ & $\mathrm{Ca}$ & Mg \\
\hline $0-10$ & 1,47 & 4,63 & 3,12 & 2058 & 2,8 & 510,2 & 1423,0 & 347,5 \\
\hline $10-30$ & 1,45 & 4,60 & 2,82 & 3770 & 3,7 & 938,7 & 1850,2 & 605,5 \\
\hline $30-60$ & 1,44 & 4,58 & 2,50 & 4752 & 2,9 & 1583,7 & 2246,4 & 601,3 \\
\hline
\end{tabular}

El material utilizado para el presente estudio fue colectado en un ensayo implantado con material genético de procedencia local obtenido de un área productora de semillas perteneciente a INTA Estación Experimental Agropecuaria Bella Vista ubicada en la provincia de Corrientes, Argentina.

En la evaluación del sotobosque la muestra estuvo conformada por 10 parcelas por tratamiento y estrato. En el estrato arbustivo se trabajó con parcelas de 2 x $1 \mathrm{~m}$ separando el material foliar y leñoso. El material herbáceo fue colectado en subparcelas de $1 \times 1 \mathrm{~m}$. En cuanto a la necromasa del piso 
forestal se colectó material de 10 subparcelas de $0,5 \times$ x 0,5 m por tratamiento, tomándose en cuenta hojas y material leñoso en distinto grado de descomposición. Todo el material colectado fue identificado, pesado a campo y secado posteriormente en estufa a $70{ }^{\circ} \mathrm{C}$ para la obtención del peso seco.

Para el estrato arbóreo, se siguió la metodología propuesta por Köhl et al. (2006). Se realizó un muestreo destructivo aleatorio estratificado, se apearon 6 individuos por cada densidad de plantación, tomando al azar 2 ejemplares por cada estrato (dominante, codominante y dominado).

Para el cálculo de biomasa se procedió al apeo, desrame y defoliado de los individuos seleccionados, procediéndose al pesaje del total de hojas y ramas mediante una balanza electrónica con una capacidad de $300 \mathrm{~kg}$ y un error de $0,02 \%$ (figura 2). Una muestra representativa del material de hojas y ramas fue secada a $70{ }^{\circ} \mathrm{C} \pm 2-3^{\circ} \mathrm{C}$ en laboratorio con el fin de estimar la biomasa de los distintos compartimentos (FASSOLA et al., 2010; SCHLEGEL et al., 2000). Para ello, se realizó el pesado húmedo y seco de la muestra para la obtención de la relación que permita la determinación de biomasa del individuo. En laboratorio se empleó una balanza analítica con capacidad de $3 \mathrm{~kg}$ y una precisión de 0,01 g. Se tomó la precaución de que cada muestra presente la debida identificación a fin de que el coeficiente de conversión obtenido para cada caso en particular pueda ser aplicado al peso total de hojas y ramas de la copa de cada individuo.

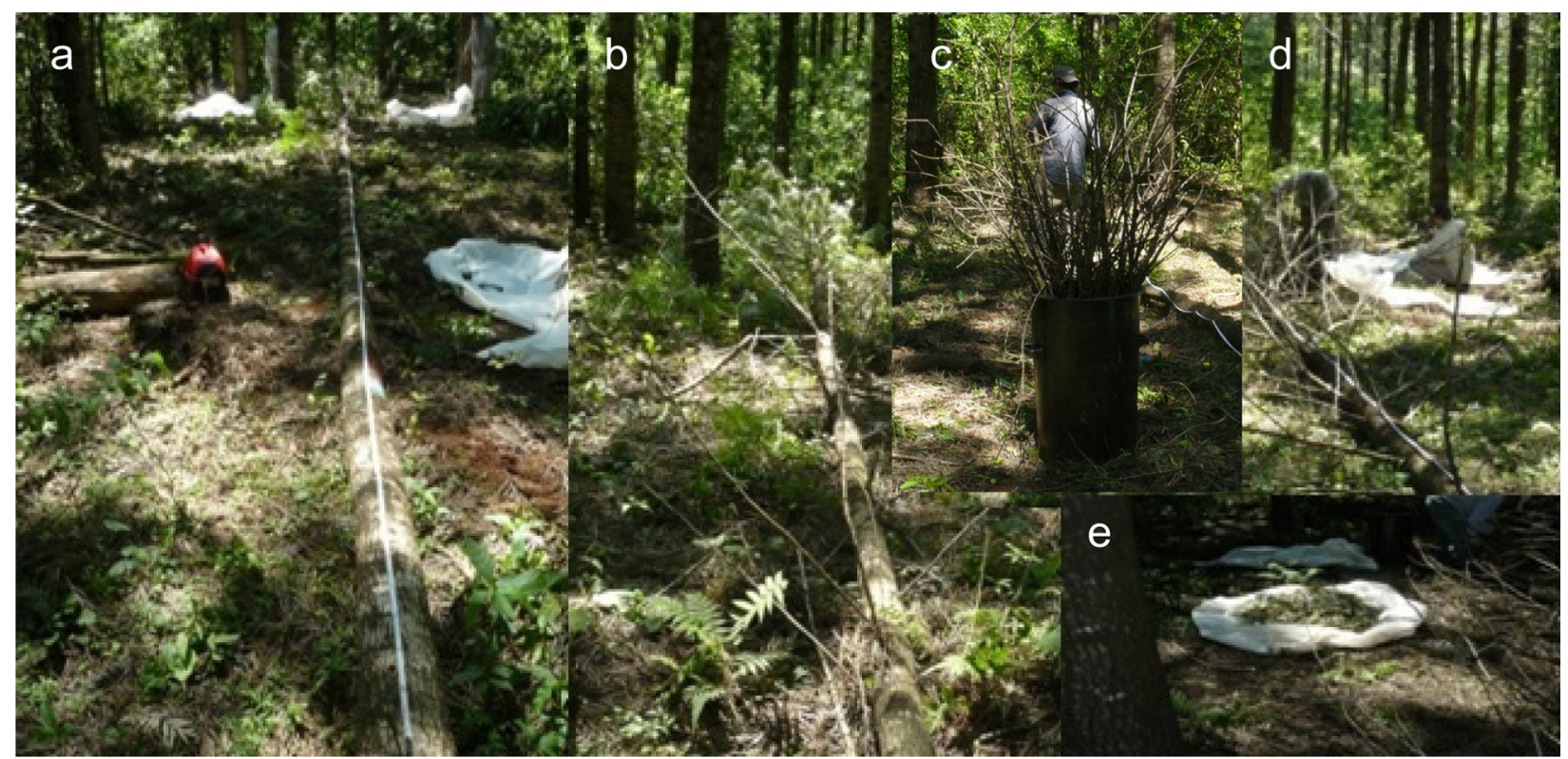

Figura 2. Apeo y desarme de individuos de Grevillea robusta. a, medición de fuste; b, trozado de copa; c, clasificación de ramas; d y e, deshoje

Figure 2. Felling and dissecting of Grevillea robusta trees. a, stem measurement; b, crown cutting; c, branches classification; $d$ and e, leaves remotion.

La biomasa de fuste fue obtenida a partir de volumetría y cálculos de densidad básica de distintas porciones del árbol (1\%, 15\%,30\%, 50\% y 80\% de su altura relativa). A partir de discos de 2 $\mathrm{cm}$ de espesor, provenientes de dichas alturas relativas, se obtuvieron probetas cúbicas de $2 \mathrm{~cm}$ de lado a partir de listones de un largo equivalente al diámetro de los discos según norma IRAM 9544 (IRAM, 1973). La densidad básica (db) se calculó como: $\mathrm{db}=\mathrm{Pa} / \mathrm{Vh}$, donde Pa es el peso anhidro y Vh es el volumen de la probeta al contenido de humedad máximo. El volumen de las probetas se calculó a través del principio de Arquímedes, ya que es considerado uno de los más prácticos y precisos. El peso anhidro de probetas se obtuvo por pesaje, tras secado a estufa hasta peso constante a una temperatura de $103 \pm 2{ }^{\circ} \mathrm{C}$. La biomasa de corteza se estableció a partir de la relación entre el porcentaje de peso de este compartimiento y el porcentaje del peso de la sección correspondiente del fuste previamente determinado (\% de corteza = peso de corteza*100/peso de fuste).

Para la comparación del efecto de la densidad de plantación en la distribución de la biomasa, se trabajó con análisis de variancia a través de modelos mixtos. Para ello se consideró la densidad de plantación como efecto fijo y a la parcela y al árbol como efecto aleatorio. En todos los casos se trabajó con test DGC con p=0,05 (Di Rienzo et al., 2002). El procesamiento se realizó a través del software InfoStat (Di Rienzo et al., 2014). 


\section{RESULTADOS Y DISCUSIÓN}

En Grevillea robusta el espaciamiento inicial tuvo efecto en el sotobosque (tabla 3) y en el crecimiento del árbol y su ahusamiento (tabla 4). Este comportamiento es citado también para Eucalyptus (SCHÖNAU; COETZEE, 1989; GERRAND et al., 1997; NEILSEN; GERRAND, 1999). Gran parte de este efecto es atribuible a la incidencia de la densidad de plantación en el microclima que se genera en la plantación, especialmente en lo relacionado a luz. El tamaño y arquitectura de la planta determinan la cantidad de luz que interceptan (CALDWELL et al., 1986; BEADLE, 1997; VALLADARES 1999). La producción de biomasa, bajo un conjunto dado de condiciones, se relaciona con la cantidad de luz interceptada por la canopia (CANNELL, 1989; KUPPERS, 1994).

La biomasa total del sotobosque se diferenció entre tratamientos $(\mathrm{P}<0,05)$ en función a la densidad de plantación, siendo significativamente menor en el tratamiento de 162 plantas por hectárea ( $\mathrm{pl} / \mathrm{ha}$ ). La presencia o ausencia de competencia da lugar a una diferenciación de la composición del sotobosque pudiendo esto ser importante en cuanto a retención de agua y nutrientes (NAMBIAR, 1985). Un caso particular se notó a $375 \mathrm{pl} /$ ha, caso analizado posteriormente en vinculación a la biomasa del estrato arbóreo (Tabla 3 ).

Tabla 3. Biomasa aérea (Mg.ha-1) acumulada en el sotobosque de Grevillea robusta en función de la densidad de plantación.

Table 3. Accumulated aboveground biomass (Mg.ha-1) at the Grevillea robusta understory based on planting density.

\begin{tabular}{lccccc}
\hline \multirow{2}{*}{ Compartimento } & \multicolumn{5}{c}{ Densidad (pl/ha) } \\
\cline { 2 - 6 } & $\mathbf{1 6 2}$ & $\mathbf{3 7 5}$ & $\mathbf{7 5 0}$ & $\mathbf{1 5 0 0}$ & Error estándar \\
\hline Arbustivo hojas & $0,31^{\mathrm{c}}$ & $0,38^{\mathrm{b}}$ & $0,28^{\mathrm{d}}$ & $0,52^{\mathrm{a}}$ & 0,05 \\
Arbustivo ramas & $0,64^{\mathrm{c}}$ & $1,21^{\mathrm{b}}$ & $2,44^{\mathrm{a}}$ & $1,08^{\mathrm{b}}$ & 0,05 \\
Herbáceo & $0,50^{\mathrm{a}}$ & $0,44^{\mathrm{b}}$ & $0,41^{\mathrm{c}}$ & $0,40^{\mathrm{c}}$ & 0,06 \\
Piso hojas & $12,05^{\mathrm{c}}$ & $14,54^{\mathrm{a}}$ & $7,58^{\mathrm{d}}$ & $12,76^{\mathrm{b}}$ & 0,36 \\
Piso ramas & $0,87^{\mathrm{c}}$ & $0,75^{\mathrm{d}}$ & $4,64^{\mathrm{a}}$ & $2,53^{\mathrm{b}}$ & 0,15 \\
Biomasa total sotobosque & $14,37^{\mathrm{c}}$ & $17,32^{\mathrm{a}}$ & $15,35^{\mathrm{b}}$ & $17,29^{\mathrm{a}}$ & 0,14 \\
\hline
\end{tabular}

Medias con una letra común no son significativamente diferentes $(\mathrm{p} \leq 0,05)$.

La diferencia en biomasa acumulada en el estrato arbustivo hojas fue estadísticamente significativa entre todos los tratamientos siendo superior a mayor densidad de plantación. En el compartimento ramas de dicho estrato las diferencias entre tratamientos no denotaron una tendencia en este sentido, aunque en la menor densidad de plantación se acumuló la menor cantidad de biomasa. En el estrato herbáceo, existieron diferencias significativas entre los tratamientos con una tendencia de mayor presencia de biomasa a menores densidades de plantación, alcanzando un valor de 0,50 $\mathrm{Mg} \mathrm{ha}^{-1} \mathrm{a} 162 \mathrm{pl} / \mathrm{ha}$. Este comportamiento puede explicarse debido a una sustitución de especies, al predominar las plantas herbáceas disminuye la presencia de especies arbustivas. La predominancia del estrato herbáceo a mayor apertura del dosel podría deberse a que a mayores espacios abiertos aparece la vegetación natural típica de la zona de campos y estepas (CABRERA, 1976). En sintonía con esta apreciación, en la Patagonia Argentina, Rusch et al. (2008) comentan que bajas coberturas arbóreas dadas por plantación de especies exóticas promueven la supervivencia y regeneración de las especies dominantes de pastizales de Festuca spp. o de bosques de Austrocedrus chilensis (ciprés de la cordillera), una especie arbórea nativa de la región. En doseles más cerrados se da un mayor número de especies arbustivas que se ven favorecidas por una mayor humedad. Esto último hace que también se pueda divisar la presencia de helechos.

En cuanto a la necromasa del piso forestal los tratamientos fueron estadísticamente diferentes entre sí. La gran acumulación de hojas en el tratamiento de mayor densidad de plantación puede deberse a elevada mortandad por competencia. La biomasa leñosa de este estrato presentó diferencias estadísticamente significativas entre todos los tratamientos destacándose su disminución a menores densidades de plantación. La acumulación de necromasa de hojas y ramas sigue la misma tendencia detectada en Pinus taeda cultivado en la zona norte de Misiones, Argentina. Martiarena et al. (2011) analizando el efecto del raleo en plantaciones de 711, 364 y 122 árboles/ha encontraron en necromasa valores de 25, 18,3 y 16,7 $\mathrm{Mg} \mathrm{ha}^{-1}$, respectivamente. La diferencia entre ambas especies se debe a características de forma y estructura propias de cada una de ellas. 
Barth et al. - Compartimentalización de la biomasa aérea de

Grevillea robusta A. en función a la densidad inicial de plantación

Considerando el estrato arbóreo, en la tabla 4 se muestran diferentes variables de estado según densidades de plantación.

Tabla 4. Variables dendrométricas del rodal de Grevillea robusta a los 18 años de edad.

Table 4. Dendrometric variables of 18-year-old Grevillea robusta stand.

\begin{tabular}{|c|c|c|c|c|}
\hline $\begin{array}{l}\text { Densidad inicial de } \\
\text { plantación (pl/ha) }\end{array}$ & $\begin{array}{c}\text { Volumen c/c } \\
\left(\mathrm{m}^{3} / \mathrm{ha}\right)\end{array}$ & $\begin{array}{c}\text { dap c/c } \\
(\mathbf{c m})\end{array}$ & $\begin{array}{c}\text { altura media de árboles } \\
\text { dominantes }(\mathrm{m})\end{array}$ & $\begin{array}{c}\text { Ahusamiento } \\
(\mathrm{cm} / \mathrm{m})\end{array}$ \\
\hline 162 & $\begin{array}{c}111,11^{\mathrm{b}} \\
(16,0)\end{array}$ & $\begin{array}{c}32,99^{a} \\
(6,4)\end{array}$ & $\begin{array}{c}18,00^{\mathrm{b}} \\
(1,9)\end{array}$ & $\begin{array}{l}1,96^{a} \\
(0,2)\end{array}$ \\
\hline 375 & $\begin{array}{c}163,93^{b} \\
(34,1)\end{array}$ & $\begin{array}{c}29,02^{b} \\
(6,1)\end{array}$ & $\begin{array}{c}20,49^{a} \\
(3,2)\end{array}$ & $\begin{array}{l}1,86^{a} \\
(0,3)\end{array}$ \\
\hline 750 & $\begin{array}{c}316,42^{a} \\
(68)\end{array}$ & $\begin{array}{c}25,44^{\mathrm{c}} \\
(5,8)\end{array}$ & $\begin{array}{c}20,60^{a} \\
(2,2)\end{array}$ & $\begin{array}{l}1,74^{a} \\
(0,3)\end{array}$ \\
\hline 1500 & $\begin{array}{c}309,03^{a} \\
(60)\end{array}$ & $\begin{array}{c}20,15 d \\
(5,3)\end{array}$ & $\begin{array}{c}20,66^{a} \\
(2,5)\end{array}$ & $\begin{array}{l}1,39^{b} \\
(0,3)\end{array}$ \\
\hline
\end{tabular}

Medias con una letra común no son significativamente diferentes $(\mathrm{p} \leq 0,05)$. Desvío estándar entre paréntesis

El dap medio fue afectado por la cantidad de árboles por hectárea con una tendencia creciente significativa hacia la menor densidad, pasando de $20,15 \mathrm{~cm}(1500 \mathrm{pl} / \mathrm{ha})$ a $32,99 \mathrm{~cm}(162 \mathrm{pl} / \mathrm{ha})$; lo que significa una diferencia de $64 \%$ entre la mayor y la menor densidad de plantación. La altura media de los árboles dominantes se mostró levemente afectada por el tratamiento silvícola aplicado. No se evidenciaron diferencias significativas entre 375 y $1500 \mathrm{pl} / \mathrm{ha}$, sí entre éstos tratamientos y $162 \mathrm{pl} / \mathrm{ha}$. Este hecho podría deberse a que la ausencia de competencia por luz hace innecesario que el individuo busque ganar altura a fin de optimizar el aprovechamiento de dicho recurso. Un comportamiento similar fue observado por Bernardo et al. (1998) en Eucalyptus camaldulensis, E. pellita y E. urophylla.

La densidad de plantación influyó significativamente en la producción volumétrica individual y del rodal. Considerando los resultados obtenidos a nivel de rodal, a mayor densidad de plantación ( $1500 \mathrm{pl} / \mathrm{ha}$ ) se obtuvo un volumen de $309,03 \mathrm{~m}^{3} / \mathrm{ha}$, lo que representa un valor superior en un 47 $\%$ y un $64 \%$ respecto a 375 y $162 \mathrm{pl} / \mathrm{ha}$. El ahusamiento fue menor en la mayor densidad de plantación, diferenciándose de los demás tratamientos. La fuerte competencia entre individuos hizo incrementar la mortalidad de éstos a mayores densidades de plantación. A los 18 años de edad, a $1500 \mathrm{pl} /$ ha la sobrevivencia fue de $68 \%$, mientras que en los demás tratamientos el valor promedio superó $80 \%$.

Individuos de mayor acumulación de biomasa fustal hicieron evidente diferencias en la biomasa del resto de los compartimentos aéreos del árbol. Se detectaron así interacciones o respuestas diferenciadas en acumulación de hojas y ramas vivas y muertas, así como también en el tamaño y acumulación de ramas (tabla 5).

Tabla 5. Biomasa aérea individual $(\mathrm{kg})$ de Grevillea robusta a 18 años de edad.

Table 5. Individual aboveground biomass $(\mathrm{kg})$ of 18-year-old Grevillea robusta.

\begin{tabular}{lccccc}
\hline \multirow{2}{*}{ Compartimento } & \multicolumn{5}{c}{ Densidad (pl/ha) } \\
\cline { 2 - 6 } & $\mathbf{1 6 2}$ & $\mathbf{3 7 5}$ & $\mathbf{7 5 0}$ & $\mathbf{1 5 0 0}$ & Error estándar \\
\hline Hojas & $17,53 \mathrm{a}$ & $16,29 \mathrm{a}$ & $10,03 \mathrm{~b}$ & $4,29 \mathrm{~b}$ & 2,7 \\
Ramas vivas $<5 \mathrm{~cm}$ & $60,40 \mathrm{a}$ & $47,91 \mathrm{a}$ & $40,03 \mathrm{a}$ & $20,38 \mathrm{~b}$ & 9,1 \\
Ramas vivas $>5 \mathrm{~cm}$ & $25,68 \mathrm{a}$ & $27,96 \mathrm{a}$ & $0,01 \mathrm{c}$ & $3,99 \mathrm{~b}$ & 5,1 \\
Ramas muertas & $0,27 \mathrm{~b}$ & $2,28 \mathrm{~b}$ & $0,43 \mathrm{~b}$ & $7,08 \mathrm{a}$ & 0,58 \\
Subtotal Ramas & $86,35 \mathrm{a}$ & $78,15 \mathrm{a}$ & $40,47 \mathrm{~b}$ & $31,45 \mathrm{~b}$ & 9,53 \\
Corteza & $38,78 \mathrm{a}$ & $25,35 \mathrm{a}$ & $28,57 \mathrm{a}$ & $13,39 \mathrm{~b}$ & 5,2 \\
Fuste & $250,24 \mathrm{a}$ & $204,21 \mathrm{a}$ & $228,40 \mathrm{a}$ & $112,06 \mathrm{~b}$ & 48,6 \\
Biomasa total & $392,90 \mathrm{a}$ & $324,00 \mathrm{a}$ & $307,47 \mathrm{a}$ & $161,19 \mathrm{~b}$ & 60,5 \\
\hline
\end{tabular}

Medias con una letra común no son significativamente diferentes $(p \leq 0,05)$

Analizando lo ocurrido a nivel de árbol individual, la densidad de plantación influyó en la partición de biomasa aérea del árbol. La biomasa total fue menor a mayor densidad de plantación. A menores densidades de plantación (162 y 375 pl/ha) la acumulación de biomasa de hojas fue mayor que a mayores densidades de plantación 750 y 1500 pl/ha). Se pudo confirmar los resultados 
obtenidos para otras especies, los cuales sugieren que a mayor densidad de plantación predominan ramas de menor diámetro (MÄKELA, 1997; NEILSEN; GERRAND, 1999). El hecho de que a menor densidad de plantación se dieran mayores diámetros de rama afecta el rendimiento de madera aserrada para los diferentes grados de calidad. Se hace entonces indispensable priorizar la realización de podas en tiempo y forma para no perjudicar el valor económico del producto a obtener. Si nuestra perspectiva es maximizar volumen de madera libre de nudos, la selección del espaciamiento inicial es de suma importancia. La competencia por recursos hizo que el tratamiento silvícola de $1500 \mathrm{pl} /$ ha evidenciara una mayor mortandad de ramas. Neilsen y Gerrand, (1999) hallaron que un incremento en la competencia entre árboles de Eucalyptus nitens tuvo influencia en la forma del fuste, sin embargo, Grevillea robusta no presentó diferencias estadísticamente significativas en ahusamiento al comparar los tratamientos de 162 a $750 \mathrm{pl} / \mathrm{ha}$ entre sí. Dicha diferencia sí surge entre éstos tratamientos y el de $1500 \mathrm{pl} / \mathrm{ha}$, en el que es evidente también una menor acumulación de biomasa total a nivel individual. Densidades de 162 a $750 \mathrm{pl} /$ ha tuvieron mayor producción individual de madera en el fuste y de corteza.

$\mathrm{Al}$ mismo tiempo que la biomasa individual tiende a incrementarse a menores densidades de plantación, la biomasa total a nivel de rodal $\left(\mathrm{Mg} \mathrm{ha}^{-1}\right)$ se incrementa a mayores densidades de plantación (tabla 6).

Tabla 6. Biomasa arbórea a nivel de rodal en Grevillea robusta a 18 años de edad. (Mg ha-1). Table 6. Stand aboveground biomass of 18 -year-old Grevillea robusta (Mg ha-1).

\begin{tabular}{lccccc}
\hline \multirow{2}{*}{ Compartimento } & \multicolumn{5}{c}{ Densidad (pl/h) } \\
\cline { 2 - 5 } & $\mathbf{1 6 2}$ & $\mathbf{3 7 5}$ & $\mathbf{7 5 0}$ & $\mathbf{1 5 0 0}$ & Error estándar \\
\hline Hojas & $3,73^{\mathrm{c}}$ & $5,05^{\mathrm{b}}$ & $8,18^{\mathrm{a}}$ & $4,89^{\mathrm{b}}$ & 0,52 \\
Ramas vivas $<5 \mathrm{~cm}$ & $8,83^{\mathrm{d}}$ & $14,11^{\mathrm{c}}$ & $23,58^{\mathrm{a}}$ & $23,13^{\mathrm{b}}$ & 0,97 \\
Ramas vivas $>5 \mathrm{~cm}$ & $10,38^{\mathrm{a}}$ & $7,68^{\mathrm{b}}$ & $0,00^{\mathrm{d}}$ & $0,17^{\mathrm{c}}$ & 0,00 \\
Ramas muertas & $0,27^{\mathrm{b}}$ & $2,28^{\mathrm{b}}$ & $0,43^{\mathrm{b}}$ & $7,08^{\mathrm{a}}$ & 0,78 \\
Subtotal Ramas & $19,48^{\mathrm{d}}$ & $24,07^{\mathrm{c}}$ & $24,01^{\mathrm{b}}$ & $30,38^{\mathrm{a}}$ & 3,32 \\
Cortez & $6,58^{\mathrm{b}}$ & $8,55^{\mathrm{b}}$ & $17,36^{\mathrm{a}}$ & $14,79^{\mathrm{a}}$ & 1,16 \\
Fuste & $49,47^{\mathrm{d}}$ & $77,75^{\mathrm{c}}$ & $127,24^{\mathrm{b}}$ & $134,44^{\mathrm{a}}$ & 4,27 \\
Biomasa total & $79,26^{\mathrm{d}}$ & $115,42^{\mathrm{c}}$ & $176,79^{\mathrm{b}}$ & $184,50^{\mathrm{a}}$ & 6,09 \\
\hline
\end{tabular}

Medias con una letra común no son significativamente diferentes $(p \leq 0,05)$

A nivel de rodal, mayores densidades de plantación tienden a acumular mayor biomasa de ramas finas por hectárea incrementándose la presencia de ramas muertas a los $1500 \mathrm{pl} / \mathrm{ha}$ debido a la competencia por recursos (luz). Por otra parte los tratamientos de 375 y $162 \mathrm{pl} /$ ha presentaron mayor biomasa de ramas gruesas por hectárea. En lo que respecta a la acumulación de biomasa en fuste a nivel de rodal, esta difirió según tratamiento y mostró una tendencia a disminuir a menores densidades de plantación. En los tratamientos de mayor densidad de plantación el número de plantas por hectárea tiene una alta incidencia en la producción total de biomasa del rodal. Se confirman así las conclusiones de Pinkard y Neilsen (2003) al estudiar Eucalyptus nitens.

Analizando la incidencia de cada compartimento aéreo en la biomasa del rodal (figura 3), el efecto de la densidad de plantación es evidente en la biomasa de fuste donde existe un incremento medio del $15 \%$ de su representatividad a favor de las mayores densidades. Si bien se presentan diferencias estadísticas significativas en todos los componentes, las tendencias no son definidas. Es destacable que a $1500 \mathrm{pl} / \mathrm{ha}$ se presenta el menor desarrollo de árbol individual, en coincidencia con la menor presencia de hojas en relación a los demás componentes aéreos del dosel.

En Grevillea robusta, la incidencia de la biomasa de hojas fue menor a la reportada para las demás especies de cultivo en Misiones, Argentina, para producción de madera. En Pinus taeda, Fassola et al. (2012) hacen referencia a valores promedio de $6 \%$ de acículas, $18 \%$ de ramas, $74 \%$ de fuste y $2 \%$ de corteza. Éste último valor denota una mayor participación de corteza en la biomasa de Grevillea robusta. Según Winck et al. (2015) en rodales de Eucalyptus grandis de la misma zona de cultivo, la biomasa foliar representa un $2 \%$, las ramas un $12 \%$ y el fuste un $86 \%$ de la biomasa total, considerándose despreciable la proporción de corteza. Este patrón diferencial de acumulación de biomasa a mayores densidades de plantación puede deberse a una diferenciación en la reacción frente a la competencia. Tal lo mencionado por MARTIARENA et al. (2008) para Pinus taeda, es probable que 
acumule mayor cantidad de biomasa en fuste para poder acceder al dosel superior en busca de luz a expensas de otros órganos o compartimentos. Esto es coincidente con que a mayores densidades de plantación se dieron las mayores alturas (tabla 4). La mayor acumulación relativa de hojas y ramas se dio en los tratamientos de 162 a $750 \mathrm{pl} /$ ha en coincidencia con el mayor dap de los individuos. El aumento de la proporción de ramas puede deberse a la necesidad de mayor capacidad portante de la copa para sostener al componente fotosintético responsable del mayor crecimiento diamétrico (HENSKENS et al., 2001).

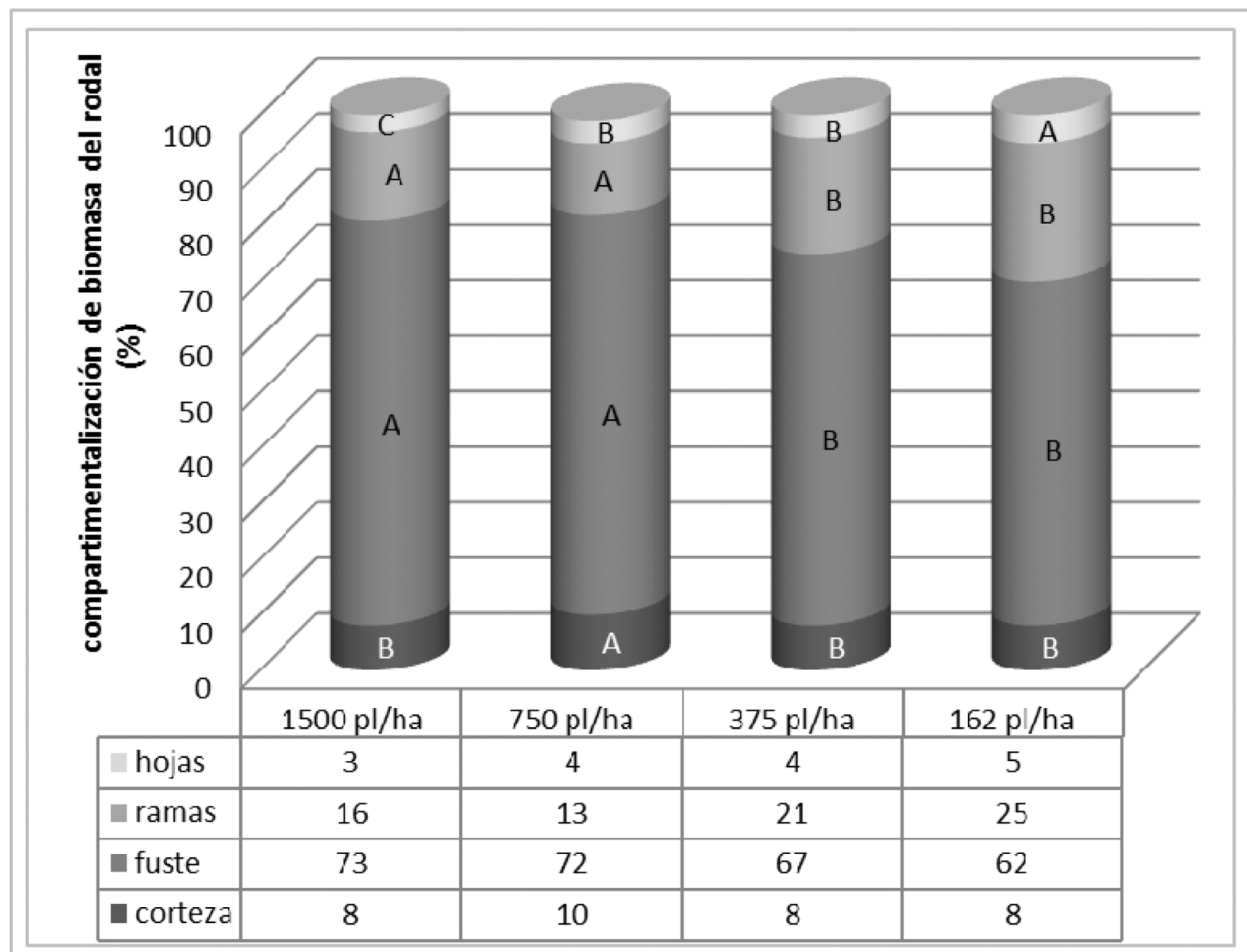

\section{Densidad de plantaclón}

medias con una letra en común no son significativamente diferentes $(p \leq 0,05)$

Figura 3. Distribución representativa de los distintos componentes de la biomasa aérea de Grevillea robusta según densidad de plantación.

Figure 3. Representative distribution of the various components of the aboveground biomass of Grevillea robusta based on planting densities.

Analizando la biomasa total por hectárea incluyendo tanto componentes arbóreos como de sotobosque, la incidencia de este último estrato presenta una tendencia creciente desde la mayor a la menor densidad de plantación. Mientras que a 1500 y 750 pl/ha alcanza un 9,37 y 8,68\% respectivamente, sin diferencia estadísticamente significativa entre ellos; a $375 \mathrm{pl} / \mathrm{ha}$ llega a 15\% y a $152 \mathrm{pl} / \mathrm{ha}$ alcanza $18 \%$. En ensayos llevados a cabo en la zona norte de Misiones en Pinus taeda, Martiarena (2008) y Martiarena et al. (2011) hallaron una menor incidencia del sotobosque en la biomasa total pero manteniendo igual comportamiento frente a las distintas densidades de plantación (a mayor cantidad de pl/ha se presenta una mayor proporción de biomasa de sotobosque). La menor acumulación de biomasa por hectárea dada en Grevillea robusta, en comparación a Pinus taeda y Eucalyptus grandis, las otras dos especies de mayor superficie cultivada en la región, se debe a diferencias de arquitectura y composición de la parte aérea de los árboles. 
Ante los resultados hallados, se recomienda que el diseño de la plantación y el posterior manejo de la masa forestal sean elegidos dependiendo del objetivo del productor. Si el productor privilegia el componente forestal, apuntando a la vez a mayores volúmenes de madera, estos se dan a mayores densidades, si desea maximizar la calidad de la madera debería considerar la conveniencia de una menor densidad final a fin de lograr trozas de mayores dimensiones. Esta última opción le daría lugar a la implementación de un sistema agroforestal combinando el desarrollo de fuste maderable de alto valor con el desarrollo del estrato herbáceo con miras a la incorporación de la producción ganadera.

\section{CONCLUSIONES}

La densidad inicial de plantación influyó en el desarrollo del sotobosque de rodales de Grevillea robusta, como en la distribución y acumulación de biomasa aérea del estrato arbóreo de los mismos. Los espaciamientos mayores dieron lugar a una menor presencia de biomasa de estrato herbáceo en el sotobosque, dicha tendencia no se mantuvo en el estrato arbustivo en donde una mayor densidad de plantación dio lugar a una mayor acumulación de biomasa. En cuanto al piso forestal se dio un mayor depósito de ramas muertas en las mayores densidades, esta tendencia no se mantuvo al considerar a las hojas muertas. Mayores espaciamientos presentaron un mayor diámetro a altura de pecho, no evidenciándose diferencias sustanciales en cuanto a altura media del rodal con excepción del tratamiento de $162 \mathrm{pl} / \mathrm{ha}$. El ahusamiento fue menor a $1500 \mathrm{pl} /$ ha diferenciándose estadísticamente de los demás tratamientos. La densidad de plantación afectó la producción de ramas. Cuanto mayor fue la densidad de plantación mayor la acumulación de ramas. Las ramas gruesas predominaron en los mayores espaciamientos. En los tratamientos de mayor densidad de plantación el fuste representó más del $70 \%$ de la biomasa total. Menores espaciamientos presentaron mayores volúmenes de madera a pesar de su menor diámetro individual.

\section{AGRADECIMIENTOS}

Los autores agradecen la colaboración en el trabajo de campo del personal de apoyo de INTA EEA Montecarlo en persona de los señores Otto Eduardo Knebel, Roberto Oscar Kobs, Lucas Giménez, Diego Aquino, Delia Sosa y Jorge Vicentini. Se contó además con la colaboración de Pamela Alarcón, Ernesto Crechi, Aldo keller, Martín Pinazo, Alejandra Von Wallis, Norberto Pahr y Eliane Romberg. El trabajo forma parte de la tesis doctoral del primer autor, siendo financiado por INTA y el PIA 10051. Componente Plantaciones Forestales Sustentables. Proyecto MSRN BIRF LN 7520 AR.

\section{REFERENCIAS BIBLIOGRÁFICAS}

ACHIM, A.; GARDINER, B.; DAQUITAINE, R. Predicting the branching properties of Sitka Spruce grown in Great Britain. New Zealand Journal of Forestry Science, Rotorua, v. 36, n. 2/3, p. 246-264, 2006.

ADEGBIDI, H. G.; JOKELA, E. J.; COMERFORD, N. B.; BARROS, N. F. Biomass development for intensively manager Loblolly pine plantations growing on Spodosols in the Southeastern USA. Forest Ecology and Management, Amsterdam, v. 167, n. 1-3, p. 91-102, 2002.

BARTH, S. R.; GIMÉNEZ, A. M.; JOSEAU, M. J.; GAUCHAT, M. E.; FASSOLA, H. E. Efecto de la densidad de plantación de Grevillea robusta A. Cunn. sobre la calidad de la madera en vistas a un uso agroforestal de la especie. IN: CONGRESO INTERNACIONAL SOBRE SISTEMAS AGROFORESTALES PARA LA PRODUCCIÓN PECUARIA Y FORESTAL SOSTENIBLE, 8. CONGRESO NACIONAL DE SISTEMAS SILVOPASTORILES, 3. Iguazú. Misiones. Argentina. Actas... Missiones, 2015. p. 243-248. CD ROOM.

BEADLE, C. Dynamics of leaf and canopy development. In: NAMBIAR E. Z.; BROWN, A. G. (Ed.) Management of soil, nutrients and water in tropical plantation forests. Canberra: ICIAR, 1997. p. 169-212.

BEAULIEU, E.; SCHNEIDER, R.; BERNINGER, F.; HUOR UNG, C.; SWIFT, E. Modeling Jack pine branch characteristics in Eastern Canada. Forest Ecology and Management, Amsterdam, v. 262, n. 9, p. 1748-1757, 2011.

Sci. For., Piracicaba, v. 44, n. 111, p. 653-664, set. 2016 DOI: dx.doi.org/10.18671/scifor.v44n111.11 
Barth et al. - Compartimentalización de la biomasa aérea de

Grevillea robusta A. en función a la densidad inicial de plantación

BERNARDO, A.; REIS, M. L.; REIS, G. F.; HARRISON, R. G.; FIRME, D. J. Effect of spacing on growth and biomass distribution in Eucalyptus camaldulensis, E. pellita, E. urophylla plantations in southeastern Brazil. Forest Ecology and management, Amsterdam, v. 104, n. 1-3, p. 1-13, 1998.

BOLAND, D. J.; BROOKER, M. I. H.; CHIPPENDALE, G. M. ; HALL, N.; HYLAND, B. P. M.; JOHNSTON, R. D.; KLEINIG, D. A.; TURNER, J. D. Forest trees of Australia. 4.ed. Melbourne: CSIRO, 1984. 687 p.

BURGOS, J. J. El clima de la región Nordeste de la República Argentina en relación con la vegetación natural y el suelo. Boletín de la Sociedad Argentina de Botánica. v. 11, p. 37-102, 1970.

CABRERA, A. L. Regiones fitogeográficas argentinas. In: KUGLER, W. F. (Ed.) Enciclopedia argentina de agricultura y jardinería. 2.ed. Buenos Aires: Acme, Tomo 2, Fascículo 1. 1976. 85 p.

CALDWELL, M.; MEISTER, H.; TENHUNEN, J.; LANGE, O. Canopy structure, light microclimate and leaf gas exchange of Quercus coccifera L. in a Portuguese macchia: measurements in different canopy layers and simulations with a canopy model. Trees, New York, v. 1, n. 1, p. 25-41, 1986.

CANNELL, M. Physiological basis of wood production: a review. Scandinavian Journal of Forerst Research, v. 4, n. 1, p. 459-490, 1989.

DIDION, M.; KAUFMANN, E.; THÜRIG, E. Impacts of forest management on carbon stock changes in litter and soil in Swiss forests. Birmensdorf: Swiss Federal Institute for Forest, Snow and Landscape Research WSL, 2014. Disponível em: <www.bafu.admin.ch/climatereporting>. Acesso em: 01 jun. 2015.

DI RIENZO J. A.; CASANOVES F.; BALZARINI M. G.; GONZALEZ L.; TABLADA M.; ROBLEDO C. W. InfoStat versión 2014. Córdoba: Grupo InfoStat, FCA, Universidad Nacional de Córdoba, Argentina, 2014. Disponível em: < http://www.infostat.com.ar $>$. Acesso em 10 jun. 2016.

DI RIENZO, J. A.; GUZMÁN, A. W.; CASANOVES, F. A. Multiple Comparisons Method based on the Distribution of the Root Node Distance of a Binary Tree. Journal of Agricultural, Biological, and Environment Statistics, v. 7, n. 2, p. 1-14, 2002.

FASSOLA, H. E.; CRECHI, E. H.; BARTH, S. R.; KELLER, A. E.; WINCK, R. A.; VIDELA, D. Análisis de las diferencias de partición de biomasa aérea entre Pinus elliottii var. elliottii x Pinus caribaea var. hondurensis (f2) y de Pinus taeda para el norte de Misiones, Argentina. In: JORNADAS TÉCNICAS FORESTALES Y AMBIENTALES, 15, 2012. Eldorado. Misiones. Argentina. Actas... Misiones: FCF UNaM- INTA, 2012. 10 p. CD ROOM.

FASSOLA, H. E.; CRECHI, E.; BARTH, S. R.; KELLER, A. E.; WINCK, R. A.; MARTIARENA, R.; VON WALLIS, A.; PINAZO, M. A.; KNEBEL, O. Modelos regionales de biomasa aérea de Pinus taeda para Misiones y NE de Corrientes, Argentina. IN: JORNADAS TÉCNICAS FORESTALES Y AMBIENTALES, 14. 2010. Eldorado. Misiones. Argentina. Actas... Misiones: FCF UNaM- INTA, 2010. 11 p. CD ROOM.

GERRAND, A. M.; NEILSEN, W. A.; MEDHURST, J. L. Thinning and pruning eucalypt plantations for sawlog production in Tasmania. Tasforests, n. 9, p. 15-34, 1997.

GONÇALVES MARTINS, E.; NEVES, E. J. M. Grevillea robusta (Cunn.): Sementes melhoradas para usos múltiplos. Colombo: Embrapa Florestas. 2004. 4 p. (Comunicado Técnico, 126)

HARWOOD, C. E. Natural distribution and ecology of Grevillea robusta. In: HARWOOD, C. E. (Ed.) GREVILLEA ROBUSTA IN AGROFORESTRY AND FORESTRY, PROCEEDINGS OF AN INTERNATIONAL WORKSHOP. 1992, Nairobi, Proceedings... Nairobi: ICRAF, 1992. p. 21-28.

HARWOOD, C. E.; GETHAUN, A. Australian tree finds success in Africa. Agroforestry Today, Nairobi, v. 2, n. 1, p. 8-10, 1990. 
HENSKENS, F. L.; BATTAGLIA, M.; CHERRY, M. L.; BEADLE, C. L. Physiological basis of spacing effects on tree growth and form in Eucalyptus globulus. Trees, New York, v. 15, n. 6, p. 365-377, 2001.

HERRERA ARANGO, M. A.; DEL VALLE, J. I.; ALONSO ORREGO, S. Biomasa de la vegetación herbácea y leñosa pequeña y necromasa en bosques tropicales primarios y secundarios de Colombia. In: SIMPOSIO INTERNACIONAL MEDICIÓN Y MONITOREO DE LA CAPTURA DE CARBONO EN ECOSISTEMAS FORESTALES. 2001. Valdivia. Chile. Actas... Valdivia, 2001. 18 p. CD ROOM.

IRAM. 9544: Norma para métodos de determinación de densidad aparente en maderas. Buenos Aires: Instituto Argentino de Racionalización de Materiales, 1973. 10 p.

JANDL, R.; ALM, J.; VESTERDAL, L.; OLSSON, M.; WEISS, P.; SJÖGERSTEN, S.; RODEGHIERO, M.; LEIFELD, J.; HAGEDORN, F.; BELLAMY, P.; BARITZ, R. Soil Carbon in Sensitive European Ecosystems: From Science to Land Management - A Summary. In: JANDL, R.; RODEGHIERO, M.; OLSSON, M. (Ed.) Soil Carbon in Sensitive European Ecosystems. Chichester: John Wiley \& Sons, Ltd. 2011. p. 267-281.

KÖHL, M.; MAGNUSSEN, S.; MARCHETTI M. Sampling Methods, Remote Sensing and GIS Multiresource Forest Inventory. Heidelberg: Springer, 2006. 374 p.

KUPPERS, M. Canopy gaps: competitive light interception and economic space filling - a matter of whole plant allocation. In: CALDWELL, M.; PEARCY, R. (Ed.) Exploitation of environmental heterogeneity by plants. San Diego: Academic Press, 1994. p. 111-144.

MÄKELA, A. A Carbon balance model of growth and self-prunning in trees based on structural relationships. Forest Science, Bethesda, v. 43, n. 1, p. 7-23, 1997.

MARILOU, B.; MESSIER, C.; LEDUC, A. Understorey light profiles in temperate deciduous forests: recovery process following selection cutting. Journal of Ecology, Oxford, v. 92, p. 328-338, 2004.

MARTIARENA, R. A. Cuantificación y evaluación del contenido de fósforo exportado en plantaciones de Pinus taeda conducido con diferentes intensidades de raleo y alternativos sistemas de cosecha. 2008. 76 p. Dissertação (Mestrado em Ciências Florestais) - Universidad Nacional de Misiones. Misiones, 2008.

MARTIARENA, R. A.; PINAZO, M. A.; VON WALLIS, A.; KNEBEL, O.; PAHR, N. M. Alternativas de manejo para la conservación de nutrientes en sistemas forestales en Misiones, Argentina. Revista de la Asociación Argentina de la Ciencia del Suelo. Buenos Aires, v. 29, n. 1, p. 39-48, 2011.

NAMBIAR, E. K. S. Critical processes in forest nutrition and their importance for management. In: LANDSBERG, J. J.; PARSONS, W. (Ed.) Research for Forest Management. Melbourne: CSIRO, 1985. p. 52-72.

NAVE, L. E.; VANCE, E. D.; SWANSTON, C. W.; CURTIS, P. S. Harvest impacts on soil carbon storage in temperate forests. Forest Ecology and Management, Amsterdam, v. 259, n. 5, p. 857-866, 2010.

NEILSEN, W. A.; GERRAND, A. M. Growth and branching habit of Eucalyptus nitens at different spacings and the effect on final crop selection. Forest Ecology and Management. Amsterdam, v. 123, n. 2-3, p. 217-229, 1999.

PRASAD, J. V. N. S.; KORWAR, G. R.; RAO, K. V.; MANDAL, U. K.; RAO, G. R.; SRINIVAS, I.; VENKATESWARLU, B.; RAO S. N.; KULKARNI H. D. Optimum stand density of Leucaena leucocephala for Wood production in Andhra Pradesh, Southern India. Biomass and bioenergy, Oxford, v. 35, n. 1, p. 227-235, 2011.

PINKARD E. A.; NEILSEN W. A. Crown and stand characteristics of Eucalyptus nitens in response to initial spacing: implications for thinning. Forest Ecology and Management, Amsterdam, v. 172, p.215-227, 2003. 
Barth et al. - Compartimentalización de la biomasa aérea de

Grevillea robusta A. en función a la densidad inicial de plantación

QUEENSLAND FOREST SERVICE. Silvicultural note on southern silky oak (Grevillea robusta). Brisbane: Anthony James Cumming, 1928. 18 p. (Bulletin, 9).

ROCHA, B.; DELLA LUCIA, R. M. Efeito do espaçamento na produçâo em peso e na qualidade da madeira de Eucalyptus grandis e Eucalyptus urophylla a os 52 meses de idade. Revista Árvore, Viçosa, v. 11, n. 2, p. 132-145, 1987.

RUSCH, V.; VILA, A.; MARQUÉS, B. Conservación de la biodiversidad en sistemas productivos: Forestaciones del Noroeste de la Patagonia. Bariloche: INTA, 2008. 89 p.

SCHLEGEL, B.; GAYOSO J.; GUERRA, J. Manual de Procedimientos Muestreos de Biomassa Forestal: Medición de la capacidad de captura de carbono en bosques de Chile y promoción en el mercado mundial. Valdivia: Universidad Austral de Chile. 2000. 24 p.

SCHÖNAU, A.; COETZEE, J. Initial spacing, stand density and thinning in Eucalypt plantations. Forest Ecology and Management, Amsterdam, v. 29, n. 4, p. 245-266, 1989.

TEOBALDELLI, M.; SOMOGYI, Z.; MIGLIAVACCA, M.; USOLTSEV, V. Generalized functions of biomass expansion factors for conifers and broadleaved by stand age, growing stock and site index. Forest Ecology and Management, Amsterdam, v. 257, n. 3, p. 1004-1013, 2009.

VALLADARES, F. Architecture, ecology and evolution of plant crowns. In: PUGNAIRE, V. (Ed.) Handbook of functional plant ecology. New York: Dekker, p. 121-194. 1999.

WANG, F.; KANG, M. Z.; LU, Q.; LETORT, V.; HAN, H.; GUO, Y.; DE REFFYE, P.; LI, B. G. A stochastic model of tree architecture and biomass partitioning: application to Mongolian Scots pines. Annals of Botany, Oxford, v. 107, p. 781-792, 2011.

WINCK, R. A.; FASSOLA, H. E.; BARTH, S. R.; CRECHI, E. H.; KELLER, A. E.; DIDELA, D.; ZADERENKO, C. Modelos predictivos de biomasa aérea de Eucalyptus grandis para el noreste de Argentina. Ciência Florestal, Santa Maria, v. 25, n. 3, p. 595-606, 2015.

ZOBEL B.; VAN BUIJTENEN, B. Wood variation: its causes and control. New York: Spring Verlag. 1989. 363 p.

Recebido em 17/04/2015

Aceito para publicação em 29/01/2016 